\title{
Melanoma brain metastasis: the impact of stereotactic radiosurgery, $B R A F$ mutational status, and targeted and/or immune-based therapies on treatment outcome
}

\author{
Rupesh Kotecha, MD, Jacob A. Miller, BS, ${ }^{2}$ Vyshak A. Venur, MD, ${ }^{3}$ Alireza M. Mohammadi, MD, ${ }^{2,4,5}$ \\ Samuel T. Chao, MD, 1,2,4 John H. Suh, MD, 1,2,4 Gene H. Barnett, MD, MBA, 2,4,5 Erin S. Murphy, MD, ,2,4 \\ Pauline Funchain, MD, ${ }^{3}$ Jennifer S. Yu, MD, PhD, ${ }^{1,2,4}$ Michael A. Vogelbaum, MD, PhD, $, 2,4$ \\ Lilyana Angelov, MD, ${ }^{2,4,5}$ and Manmeet S. Ahluwalia, MD ${ }^{2-4}$
}

\begin{abstract}
Departments of ${ }^{1}$ Radiation Oncology and ${ }^{3} \mathrm{Hematology} /$ Oncology, Taussig Cancer Institute, Cleveland Clinic; ${ }^{2}$ Cleveland Clinic Lerner College of Medicine of Case Western Reserve University; ${ }^{4}$ Rose Ella Burkhardt Brain Tumor and Neuro-Oncology Center; and ${ }^{5}$ Department of Neurosurgery, Neurological Institute, Cleveland Clinic, Cleveland, Ohio
\end{abstract}

\begin{abstract}
OBJECTIVE The goal of this study was to investigate the impact of stereotactic radiosurgery (SRS), BRAF status, and targeted and immune-based therapies on the recurrence patterns and factors associated with overall survival (OS) among patients with melanoma brain metastasis (MBM).
\end{abstract}

METHODS A total of 366 patients were treated for $1336 \mathrm{MBMs}$; a lesion-based analysis was performed on $793 \mathrm{SRS}$ lesions. The BRAF status was available for 78 patients: 35 had BRAFmut and 43 had BRAF wild-type (BRAF-WT) lesions. The Kaplan-Meier method evaluated unadjusted OS; cumulative incidence analysis determined the incidences of local failure (LF), distant failure, and radiation necrosis (RN), with death as a competing risk.

RESULTS The 12-month OS was $24 \%$ (95\% Cl 20\%-29\%). On multivariate analysis, younger age, lack of extracranial metastases, better Karnofsky Performance Status score, and fewer MBMs, as well as treatment with BRAF inhibitors (BRAFi), anti-PD-1/CTLA-4 therapy, or cytokine therapy were significantly associated with OS. For patients who underwent SRS, the 12-month LF rate was lower among those with $B R A F^{\text {mut }}$ lesions $(6 \%, 95 \% \mathrm{Cl} 2 \%-11 \%)$ compared with those with BRAF-WT lesions $(22 \%, 95 \% \mathrm{Cl} 13 \%-32 \%$; $p<0.01)$. The 12 -month $\mathrm{LF}$ rates among lesions treated with BRAFi and PD-1/CTLA-4 agents were $1 \%(95 \% \mathrm{Cl} 1 \%-4 \%)$ and $7 \%(95 \% \mathrm{Cl} 1 \%-13 \%)$, respectively. On multivariate analysis, BRAF inhibition within 30 days of SRS was protective against $\mathrm{LF}(\mathrm{HR} 0.08,95 \% \mathrm{Cl} 0.01-0.55 ; p=0.01)$. The 12-month rates of RN were low among lesions treated with BRAFi ( $0 \%, 95 \% \mathrm{Cl} 0 \%-0 \%)$, PD-1/CTLA-4 inhibitors ( $2 \%$, $95 \% \mathrm{Cl} 1 \%-5 \%$ ), and cytokine therapies (6\%, $95 \% \mathrm{Cl} 1 \%-13 \%)$.

CONCLUSIONS Prognostic schema should incorporate BRAFi or immunotherapy status and use of targeted therapies. Treatment with a BRAF inhibitor within 4 weeks of SRS improves local control without an increased risk of RN.

https://thejns.org/doi/abs/10.3171/2017.1.JNS162797

KEY WORDS brain metastasis; stereotactic radiosurgery; BRAF; vemurafenib; ipilimumab; prognostic; radiation necrosis; oncology

$\mathrm{P}$ ATIENTS with metastatic melanoma have the highest predisposition for developing brain metastasis, and autopsy studies have demonstrated brain metastases in up to $55 \%-75 \%$ of patients with metastatic melanoma. ${ }^{28}$ Melanoma is associated with a number of somatic mutations and aberrations, the most common of which is the BRAF mutation. ${ }^{22}$ The BRAF inhibitors (BRAFi), in- cluding vemurafenib and dabrafenib, and immune-based therapies, including ipilimumab, have demonstrated intracranial efficacy and improved survival in Phase II studies in patients with active brain metastasis. ${ }^{14,17}$ Furthermore, although patients in the past were treated with whole-brain radiation therapy (WBRT), recent series of patients with melanoma brain metastasis (MBM) treated with stereo-

ABBREVIATIONS BRAFi = BRAF inhibitors; $\mathrm{DF}=$ distant failure; $\mathrm{GPA}=$ graded prognostic assessment; $\mathrm{KPS}=$ Karnofsky Performance Status; $\mathrm{LF}=$ local failure; $\mathrm{MBM}=$ melanoma brain metastasis; OS = overall survival; RN = radiation necrosis; $S R S$ = stereotactic radiosurgery; WBRT = whole-brain radiation therapy; WT = wild type. SUBMITTED November 6, 2016. ACCEPTED January 26, 2017.

INCLUDE WHEN CITING Published online August 11, 2017; DOI: 10.3171/2017.1.JNS162797. 
tactic radiosurgery (SRS) have reported impressive local control rates., ${ }^{4,12,15,19}$ The impact and interplay of all these factors have yet to be empirically demonstrated in a large series of patients with MBM. Therefore, in this report we examine the natural history, intracranial recurrence patterns, and factors associated with improved survival for patients with MBM, with a specific emphasis on the study of SRS, BRAF mutational status, and the impact of targeted and immune-based therapies.

\section{Methods}

\section{Patient Selection and Data Collection}

All patients in whom MBM was diagnosed between 1987 and 2014 at a tertiary care institution were included. The following data were recorded in an institutional review board-approved institutional registry: age, sex, Karnofsky Performance Status (KPS) score, number of brain metastases, presence of extracranial metastases, graded prognostic assessment (GPA), and treatment details. For patients who underwent SRS, the prescription dose, lesion laterality, location, maximum diameter, metastasis volume, conformity index, heterogeneity index, and gradient index were also recorded. For a subset of patients diagnosed primarily after 2009, BRAF V600E mutational testing was performed.

\section{Treatment Delivery}

At the discretion of the treating physician, patients underwent a variety of treatments, including WBRT, surgery, and/or SRS. Considerations of the initial treatment approach were made based on the following variables: patient KPS score; number of brain metastases; size of the lesion(s); location of the lesion(s); neurological status of the patients and deficits; age of the patient; primary tumor extent, disease, or stage; extracranial disease progression; and patient input. Patients who underwent SRS were treated with a linear accelerator-based platform between 1987 and 1997, after which patients underwent Gamma Knife (Elekta Instruments AB) radiosurgery. For radiosurgical planning, the dose prescribed to the peripheral margin was typically chosen based on the maximum lesion size according to the Radiation Therapy Oncology Group (RTOG) protocol 90-05. ${ }^{27}$

\section{Outcome Variables}

The primary outcome was overall survival (OS), whereas secondary outcomes included the cumulative incidences of local failure (LF), distant failure (DF), and radiation necrosis $(\mathrm{RN})$. The OS was calculated from the time of the first brain metastasis, whereas secondary outcomes were calculated from the time of treatment. Patients undergoing SRS underwent imaging approximately 4-8 weeks following treatment, and at 3-month intervals subsequently. Radiographic data, including CT and MRI scans, were reviewed to determine intracranial patterns of failure. Unidirectional or bidirectional measurements were made on lesions that increased in size following treatment. Local failures were diagnosed using a range of modalities including serial imaging, MR perfusion, $\left[{ }^{18} \mathrm{~F}\right]$ fluorodeoxyglucose avidity on PET, and pathological findings, as available. In the event of disagreement among modalities, either serial imaging follow-up or multidisciplinary tumor board review was used to identify LFs. ${ }^{20}$ Similarly, RN was diagnosed using an institutional algorithm..$^{5}$ In general, ring-enhancing lesions were suggestive of $\mathrm{RN}$, but a final determination was made based on short-term followup and advanced imaging studies, including brain PET, MRI with cerebral blood volume, or surgical evaluation (biopsy or resection). ${ }^{18}$ When available, the Social Security Death Index was used to verify dates of death.

\section{Statistical Analysis}

Data were analyzed on a per-patient (OS, DF) and perlesion (LF, RN) basis. Baseline continuous data were compared via t-tests or Wilcoxon rank-sum tests. Categorical data were compared via Fisher exact tests. The KaplanMeier method was used to determine unadjusted OS, and cumulative incidence analysis was used to determine the incidences of LF, DF, and RN, with death as a competing risk. Differences in OS and secondary outcomes were compared with log-rank and Gray's tests, respectively. Multivariate Cox proportional hazards regression was used to model OS, and multivariate competing-risks regression was used to model LF, DF, and RN, with death as a competing risk. Covariates demonstrating association $(\mathrm{p}$ $\leq 0.10$ ) with primary and secondary outcomes on univariate analysis were evaluated in a multivariate model including 2-way interactions. Covariate inclusion into the final model required $\mathrm{p} \leq 0.10$. Analyses were conducted using the R statistical software package (R Core Team 2015; R Foundation for Statistical Computing). Two-sided tests with $\mathrm{p}<0.05$ were considered statistically significant.

\section{Results}

\section{Patient and Treatment Characteristics: Pooled Cohort}

The baseline characteristics of the 366 patients treated for 1336 brain metastases are presented in Table 1 . The $B R A F$ V600E mutational status was available for 78 patients (21\%): 35 patients were positive for the $B R A F$ V600E mutation $\left(B R A F^{m u t}\right)$, and 43 patients had the $B R A F$ wild-type $(B R A F-W T)$ gene. The median time from primary cancer diagnosis to brain metastasis was 31 months (range 0-382 months), with 72 patients (20\%) diagnosed with brain metastasis at presentation. Compared with patients who had $B R A F-W T$ lesions, those with $B R A F^{\text {mut }}$ tumors were younger (median age 60 vs 64 years), presented with brain metastasis at a later date from primary diagnosis (37 vs 13 months), and more often presented with hemorrhagic brain metastasis ( $31 \%$ vs $26 \%)$. Initial treatment patterns also significantly differed among $B R A F$ cohorts: those with $B R A F^{m u t}$ tumors were least likely to receive upfront WBRT compared with those who had $B R A F-W T$ or $B R A F$-unknown lesions ( $37 \%$ vs $51 \%$ vs $63 \%, \mathrm{p}<0.01)$, and were most likely to receive upfront SRS ( $71 \%$ vs $58 \%$ vs $46 \%, p=0.01$ ). No significant differences in other key characteristics, including sex, presence of extracranial metastases, KPS score, number of brain metastases, or GPA class, were observed. Following diagnosis with MBM, 22 of the 35 patients with $B R A F^{m u t}$ tumors received BRAFi, 32 patients received immunotherapies (PD-1 or CTLA- 
TABLE 1. Characteristics at presentation of 366 patients with brain metastasis

\begin{tabular}{|c|c|c|c|c|c|}
\hline \multirow[b]{2}{*}{ Characteristic } & \multicolumn{4}{|c|}{ Cohort } & \multirow{2}{*}{$\begin{array}{c}p \\
\text { Value }\end{array}$} \\
\hline & All Patients & $B R A F+$ & $B R A F-W T$ & BRAF-Unknown & \\
\hline No. patients & 366 & 35 & 43 & 288 & \\
\hline Age in yrs, mean \pm SD & $57 \pm 14$ & $60 \pm 13$ & $64 \pm 13$ & $56 \pm 14$ & $<0.01$ \\
\hline$<50$ & $94(33 \%)$ & $7(20 \%)$ & $6(14 \%)$ & $94(33 \%)$ & \multirow{3}{*}{0.03} \\
\hline $50-59$ & $104(29 \%)$ & $10(29 \%)$ & $11(26 \%)$ & $83(29 \%)$ & \\
\hline$\geq 60$ & $155(42 \%)$ & $18(51 \%)$ & $26(60 \%)$ & $111(38 \%)$ & \\
\hline Male & $227(62 \%)$ & $23(66 \%)$ & $27(63 \%)$ & $186(65 \%)$ & 0.20 \\
\hline Extracranial metastasis & $288(79 \%)$ & $30(86 \%)$ & $37(86 \%)$ & $221(77 \%)$ & 0.23 \\
\hline KPS score & $80[30-100]$ & $90[50-100]$ & $80[40-100]$ & 80 [30-100] & 0.75 \\
\hline \multicolumn{6}{|l|}{$<70$} \\
\hline \multicolumn{6}{|l|}{$70-80$} \\
\hline \multicolumn{6}{|l|}{$90-100$} \\
\hline Brain metastases & $2[1-20]$ & $2[1-15]$ & $2[1-20]$ & $2[1-20]$ & 0.95 \\
\hline 1 & $126(34 \%)$ & $13(37 \%)$ & $14(33 \%)$ & $99(35 \%)$ & \multirow{3}{*}{0.93} \\
\hline $2-3$ & $117(32 \%)$ & $9(26 \%)$ & $15(34 \%)$ & $93(32 \%)$ & \\
\hline$>3$ & $123(34 \%)$ & $13(37 \%)$ & $14(33 \%)$ & $96(33 \%)$ & \\
\hline Hemorrhagic metastasis & $62(17 \%)$ & $11(31 \%)$ & $11(26 \%)$ & $60(21 \%)$ & $<0.01$ \\
\hline GPA group & & & & & 0.10 \\
\hline $0-1$ & $107(29 \%)$ & $14(40 \%)$ & $18(42 \%)$ & $75(26 \%)$ & \\
\hline $1.5-2.5$ & $200(55 \%)$ & $16(46 \%)$ & $21(49 \%)$ & $163(56 \%)$ & \\
\hline 3 & $36(10 \%)$ & $5(14 \%)$ & $3(7 \%)$ & $28(10 \%)$ & \\
\hline $3.5-4$ & $23(6 \%)$ & $0(0 \%)$ & $1(2 \%)$ & $2(8 \%)$ & \\
\hline \multicolumn{6}{|l|}{$B R A F$ status } \\
\hline BRAF V600E+ & $35(10 \%)$ & $35(100 \%)$ & $0(0 \%)$ & $0(0 \%)$ & \\
\hline$B R A F$ V600E- & $43(12 \%)$ & $0(0 \%)$ & $43(100 \%)$ & $0(0 \%)$ & \\
\hline Unknown & $288(79 \%)$ & $0(0 \%)$ & $0(0 \%)$ & $288(100 \%)$ & \\
\hline \multicolumn{6}{|l|}{ Upfront therapy } \\
\hline WBRT & $217(59 \%)$ & $13(37 \%)$ & $22(51 \%)$ & $182(63 \%)$ & $<0.01$ \\
\hline SRS & $182(50 \%)$ & $25(71 \%)$ & $25(58 \%)$ & $132(46 \%)$ & 0.01 \\
\hline Surgery & $94(26 \%)$ & $9(26 \%)$ & $11(26 \%)$ & $93(32 \%)$ & 0.41 \\
\hline Immune or targeted therapy ${ }^{*}$ & $59(16 \%)$ & $27(77 \%)$ & $18(42 \%)$ & $14(5 \%)$ & $<0.01$ \\
\hline BRAFi & $22(6 \%)$ & $22(63 \%)$ & $0(0 \%)$ & $0(0 \%)$ & $<0.01$ \\
\hline PD-1/CTLA-4 inhibitor & $32(9 \%)$ & $14(40 \%)$ & $16(37 \%)$ & $2(1 \%)$ & $<0.01$ \\
\hline Cytokine therapy $\dagger$ & $16(4 \%)$ & $1(3 \%)$ & $3(7 \%)$ & $12(4 \%)$ & 0.64 \\
\hline Time to diagnosis of brain metastasis & $31[0-382]$ & $37[0-145]$ & $13[0-177]$ & $26[0-382]$ & 0.04 \\
\hline$\leq 12$ mos & $122(33 \%)$ & $11(31 \%)$ & $20(47 \%)$ & $93(32 \%)$ & 0.18 \\
\hline$>12$ mos & $244(67 \%)$ & $24(69 \%)$ & $23(53 \%)$ & $195(68 \%)$ & \\
\hline
\end{tabular}

4 inhibitors), and 16 patients were treated with cytokine therapy (interleukin-2 or interferon- $\alpha$ ).

The median follow-up times from primary cancer diagnosis and brain metastasis were 34 months (range 1-477 months) and 5 months (range 1-327 months), respectively. In total, 175 patients (48\%) suffered from intracranial progression following upfront therapy: 116 patients (32\%) experienced an LF and 144 patients (39\%) experienced a DF (some had both). The 6- and 12-month cumulative incidences of intracranial failure were $38 \%$ (95\% CI $33 \%-43 \%)$ and $44 \%(95 \%$ CI 39\%-49\%), respectively.
Among patients with $B R A F^{m u t}, B R A F-W T$, and $B R A F$ unknown tumors, the 12-month cumulative incidences of intracranial failure were $58 \%$ (95\% CI 41\%-76\%), $43 \%$ (95\% CI $28 \%-59 \%$ ), and $42 \%$ (95\% CI 36\%-48\%) (Fig. 1A, $\mathrm{p}=0.34$ ), respectively. Intracranial progression among patients with $B R A F^{m u t}$ lesions was primarily driven by DF compared with the other cohorts (Fig. 1B) (Table 2 ). The median OS among all patients was 6 months. The $6-, 12-$, and 18-month survival estimates were 50\% (95\% CI $45 \%-55 \%), 24 \%$ (95\% CI 20\%-29\%), and $17 \%$ (95\% CI $13 \%-21 \%$ ), respectively. The median survival for pa- 

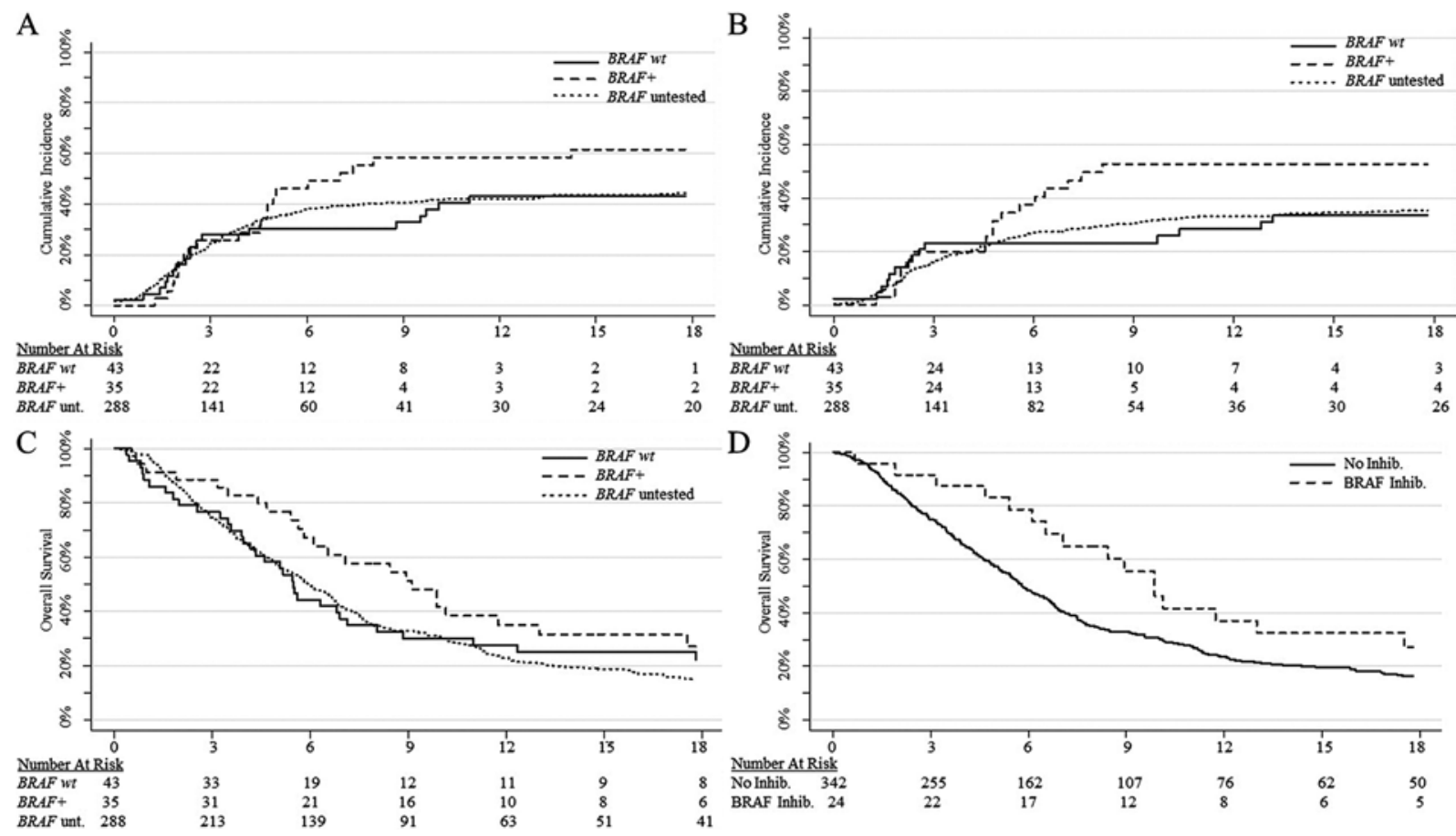

FIG. 1. Graphs showing estimates of (A) cumulative incidence of intracranial progression; (B) cumulative incidence of distant intracranial progression; and OS from the time of diagnosis of brain metastasis for patients with melanoma, stratified by (C) BRAF mutational status and (D) use of BRAFi. Values on the $\mathrm{x}$ axis denote the number of months postdiagnosis. inhib. $=$ inhibitor; unt. = untested.

tients with $B R A F^{m u t}$ tumors (9 months, $95 \%$ CI 6-13) was greater than for the $B R A F-W T$ (5 months, $95 \%$ CI 4-7) and $B R A F$-unknown cohorts (6 months, 95\% CI 5-7) (p $=0.12)($ Fig. $1 \mathrm{C})$.

Characteristics associated ( $\mathrm{p} \leq 0.10)$ with OS on univariate analysis included younger age, female sex, lack of extracranial metastases, higher KPS score, fewer brain metastases at presentation, nonhemorrhagic metastases, and treatment with BRAFi, immunotherapy, or cytokine therapy (Table 3). On multivariate analysis, younger age, lack of extracranial metastases, KPS score, number of metastases at presentation, and treatment with BRAFi and immunotherapies remained significantly associated with OS. The 6- and 12-month survival estimates were higher among patients treated with BRAFi $(79 \%, 95 \%$ CI $58 \%-91 \%$ and $37 \%, 95 \%$ CI $20 \%-58 \%$ ) compared with patients who did not receive these agents $(47 \%, 95 \%$ CI $41 \%-52 \%$ and $23 \%, 95 \%$ CI $19 \%-28 \%$ ), respectively (p $=0.01)$ (Fig. 1D). Similarly, the 6- and 12-month survival estimates among patients treated with immunotherapies were $76 \%$ (95\% CI 57\%-88\%) and 47\% (95\% CI 29\%$65 \%$ ) compared with $47 \%$ (95\% CI $42 \%-52 \%$ ) and $22 \%$ (95\% CI 18\%-27\%) among those who did not receive those therapies $(\mathrm{p}=0.04)$. Finally, the 6 - and 12 -month

TABLE 2. Unadjusted clinical outcomes in 366 patients with brain metastasis

\begin{tabular}{|c|c|c|c|c|c|}
\hline \multirow[b]{2}{*}{ Outcome } & \multicolumn{4}{|c|}{ Cohort } & \multirow{2}{*}{$\begin{array}{c}p \\
\text { Value }\end{array}$} \\
\hline & All Patients & $B R A F+$ & $B R A F-W T$ & BRAF-Unknown & \\
\hline No. of patients & 366 & 35 & 43 & 288 & \\
\hline Distant progression & $144(39 \%)$ & $18(51 \%)$ & $14(33 \%)$ & $112(39 \%)$ & 0.23 \\
\hline 6-mo & $28 \%[23 \%-32 \%]$ & $35 \%[19 \%-52 \%]$ & $23 \%[10 \%-36 \%]$ & $27 \%[22 \%-32 \%]$ & \multirow{2}{*}{0.27} \\
\hline 12-mo & $34 \%[29 \%-57 \%]$ & $53 \%[35 \%-70 \%]$ & $28 \%[14 \%-42 \%]$ & $33 \%[28 \%-39 \%]$ & \\
\hline Local or distant progression & $176(48 \%)$ & $22(63 \%)$ & $18(42 \%)$ & $136(47 \%)$ & 0.15 \\
\hline $6-\mathrm{mo}$ & $38 \%[33 \%-43 \%]$ & $46 \%[29 \%-63 \%]$ & $30 \%[16 \%-44 \%]$ & $38 \%[32 \%-44 \%]$ & \multirow{2}{*}{0.34} \\
\hline $12-\mathrm{mo}$ & $44 \%[39 \%-49 \%]$ & $58 \%[41 \%-76 \%]$ & $43 \%[28 \%-59 \%]$ & $42 \%[36 \%-48 \%]$ & \\
\hline \multicolumn{6}{|l|}{ OS } \\
\hline 6-mo & $50 \%[45 \%-55 \%]$ & $67 \%[50 \%-81 \%]$ & $44 \%[30 \%-59 \%]$ & $49 \%[44 \%-55 \%]$ & \\
\hline 12-mo & $24 \%[20 \%-29 \%]$ & $35 \%[20 \%-53 \%]$ & $28 \%[16 \%-43 \%]$ & $23 \%[18 \%-28 \%]$ & 0.12 \\
\hline 18-mo & $17 \%[13 \%-21 \%]$ & $27 \%[14 \%-46 \%]$ & $22 \%[12 \%-37 \%]$ & $15 \%[11 \%-20 \%]$ & \\
\hline
\end{tabular}

Values are presented as the number (percent) or median [range]. 
TABLE 3. Multivariate Cox proportional hazards and competing-risk regressions

\begin{tabular}{|c|c|c|c|c|c|c|c|c|c|c|c|c|}
\hline \multirow[b]{3}{*}{ Covariate } & \multicolumn{4}{|c|}{ OS } & \multicolumn{4}{|c|}{ Local Progression } & \multicolumn{4}{|c|}{ RN } \\
\hline & \multirow{2}{*}{$\begin{array}{c}\text { Univariate } \\
p \\
\text { Value }\end{array}$} & \multicolumn{3}{|c|}{ Multivariate } & \multirow{2}{*}{$\begin{array}{c}\text { Univariate } \\
p \\
\text { Value }\end{array}$} & \multicolumn{3}{|c|}{ Multivariate } & \multirow{2}{*}{$\begin{array}{c}\text { Univariate } \\
p \\
\text { Value }\end{array}$} & \multicolumn{3}{|c|}{ Multivariate } \\
\hline & & $\mathrm{HR}$ & $\begin{array}{c}95 \% \\
\mathrm{Cl}\end{array}$ & $\begin{array}{c}p \\
\text { Value }\end{array}$ & & $\mathrm{HR}$ & $\begin{array}{c}95 \% \\
\mathrm{Cl}\end{array}$ & $\begin{array}{c}p \\
\text { Value }\end{array}$ & & $\mathrm{HR}$ & $\begin{array}{c}95 \% \\
\mathrm{Cl}\end{array}$ & $\begin{array}{c}p \\
\text { Value }\end{array}$ \\
\hline \multicolumn{13}{|l|}{ Age in yrs } \\
\hline$<50$ & $<0.01$ & 0.71 & $0.54-0.92$ & 0.01 & $<0.01$ & 1.55 & $0.96-2.49$ & 0.07 & 0.12 & 1.82 & $0.86-3.82$ & 0.12 \\
\hline $50-59$ & $<0.01$ & 0.64 & $0.49-0.83$ & $<0.01$ & $<0.01$ & 1.59 & $0.99-2.57$ & 0.06 & 0.04 & 1.92 & $0.91-4.06$ & 0.09 \\
\hline$\geq 60$ & Ref & 1.00 & Ref & Ref & Ref & 1.00 & Ref & Ref & Ref & 1.00 & Ref & Ref \\
\hline Male & $<0.01$ & 1.00 & $0.58-1.82$ & 0.99 & 0.47 & - & - & - & 0.54 & - & - & - \\
\hline Extracranial metastasis & $<0.01$ & 0.61 & $0.46-0.80$ & $<0.01$ & 0.20 & - & - & - & $<0.01$ & 2.15 & $1.09-4.24$ & 0.03 \\
\hline \multicolumn{13}{|l|}{ KPS score } \\
\hline$<70$ & Ref & 1.00 & Ref & Ref & Ref & - & - & - & Ref & - & - & - \\
\hline $70-80$ & $<0.01$ & 0.36 & $0.26-0.52$ & $<0.01$ & 0.72 & - & - & - & 0.46 & - & - & - \\
\hline $90-100$ & $<0.01$ & 0.29 & $0.20-0.42$ & $<0.01$ & 0.77 & - & - & - & 0.50 & - & - & - \\
\hline \multicolumn{13}{|l|}{ No. of brain metastases } \\
\hline 1 & $<0.01$ & 0.52 & $0.39-0.69$ & $<0.01$ & $<0.01$ & 1.82 & $1.10-3.02$ & 0.02 & 0.49 & - & - & - \\
\hline $2-3$ & 0.02 & 0.69 & $0.52-0.90$ & $<0.01$ & 0.04 & 1.45 & $0.91-2.32$ & 0.02 & 0.94 & - & - & - \\
\hline$>3$ & Ref & 1.00 & Ref & Ref & Ref & 1.00 & Ref & Ref & Ref & - & - & - \\
\hline Hemorrhagic metastasis & 0.09 & 1.14 & $0.81-1.58$ & 0.72 & 0.50 & - & - & - & 0.90 & - & - & - \\
\hline \multicolumn{13}{|l|}{$B R A F$ status } \\
\hline BRAF V600E+ & 0.31 & - & - & - & $<0.01$ & 0.32 & $0.15-1.71$ & 0.81 & 0.03 & 0.27 & $0.07-0.84$ & 0.02 \\
\hline BRAF V600E- & Ref & - & - & - & Ref & 1.00 & Ref & Ref & Ref & 1.00 & Ref & Ref \\
\hline Unknown & 0.70 & - & - & - & 0.04 & 0.52 & $0.31-1.85$ & 0.24 & 0.26 & 0.36 & $0.15-1.05$ & 0.06 \\
\hline \multicolumn{13}{|l|}{ Immune or targeted therapy* } \\
\hline BRAFi & 0.01 & 0.48 & $0.28-0.92$ & $<0.01$ & $<0.01$ & 0.08 & $0.01-0.55$ & 0.01 & $\dagger$ & - & - & - \\
\hline PD-1/CTLA-4 inhibitor & 0.04 & 0.67 & $0.44-0.99$ & 0.04 & 0.03 & 0.81 & $0.38-1.75$ & 0.59 & 0.10 & 0.25 & $0.04-1.70$ & 0.15 \\
\hline Cytokine therapy & $<0.01$ & 0.54 & $0.28-0.92$ & 0.02 & 0.10 & 0.42 & $0.12-1.44$ & 0.17 & 0.82 & - & - & - \\
\hline \multicolumn{13}{|l|}{ Laterality } \\
\hline $\mathrm{Lt}$ & - & - & - & - & Ref & - & - & - & Ref & - & - & - \\
\hline Rt & - & - & - & - & 0.54 & - & - & - & 0.61 & - & - & - \\
\hline Midline & - & - & - & - & 0.38 & - & - & - & 0.48 & - & - & - \\
\hline Supratentorial & - & - & - & - & 0.99 & - & - & - & 0.26 & - & - & - \\
\hline Prior or concurrent WBRT & - & - & - & - & 0.10 & 1.10 & $0.75-1.62$ & 0.61 & 0.23 & - & - & - \\
\hline Prior or concurrent surgery & - & - & - & - & $<0.01$ & 1.22 & $0.81-1.85$ & 0.33 & 0.03 & 1.50 & $0.70-3.21$ & 0.30 \\
\hline Maximum diameter (cm) & - & - & - & - & $<0.01$ & 1.34 & $1.15-1.57$ & $<0.01$ & 0.54 & - & - & - \\
\hline Prescription dose (Gy) & - & - & - & - & $<0.01$ & 0.98 & $0.90-1.05$ & 0.53 & 0.37 & - & - & - \\
\hline Maximum dose (Gy) & - & - & - & - & 0.07 & 0.99 & $0.97-1.02$ & 0.56 & $<0.01$ & 1.03 & $1.00-1.08$ & 0.09 \\
\hline Conformity index & - & - & - & - & 0.01 & 0.81 & $0.65-1.00$ & 0.05 & 0.23 & - & - & - \\
\hline Homogeneity index & - & - & - & - & 0.69 & - & - & - & $<0.01$ & 2.25 & $0.97-5.21$ & 0.06 \\
\hline Gradient index & - & - & - & - & 0.13 & - & - & - & 0.14 & - & - & - \\
\hline
\end{tabular}

Ref $=$ reference; $-=$ not analyzed (either for that end point if it did not correlate with the outcome measure, or if nonsignificant on univariate analysis).

* Following brain metastasis.

$\dagger$ No RN events.

survival estimates among patients treated with cytokine therapies were $74 \%(95 \%$ CI $47 \%-90 \%)$ and $54 \%(95 \%$ CI $29 \%-76 \%$ ) compared with $48 \%$ (95\% CI $42 \%-53 \%$ ) and $23 \%$ (95\% CI 18\%-27\%) among those who did not receive those therapies $(p=0.01)$. Patients treated with either $\mathrm{BRAFi}$, immunotherapies, or cytokine therapies did not significantly differ from each other with respect to survival $(\mathrm{p}=0.35)$.

\section{Treatment Characteristics in Patients Receiving SRS}

A subset of patients (191 patients, 52\%) underwent SRS for 793 brain metastases during the course of their disease: 19 patients (10\%) had BRAF $F^{\text {ut }}, 24$ (13\%) had $B R A F$ $W T$, and $148(77 \%)$ had $B R A F$-unknown tumors. The median follow-up for this subset of patients was 7 months (range 1-174 months). Among the 793 treated metastases, 111 (14\%) were $B R A F^{m u t}, 77$ (10\%) were $B R A F-W T$, and 
A

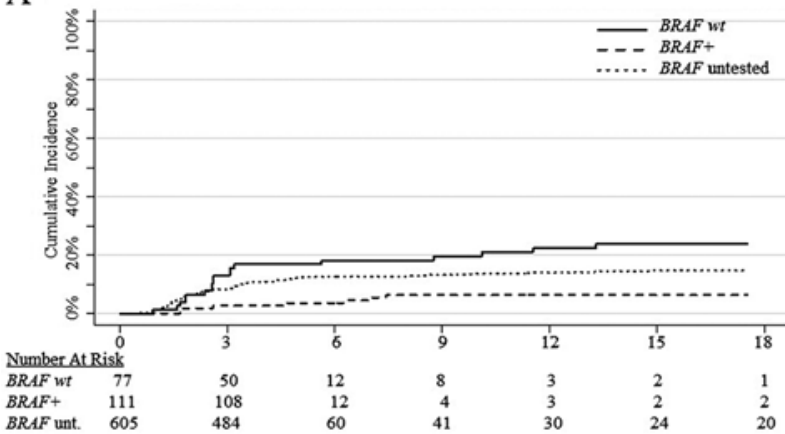

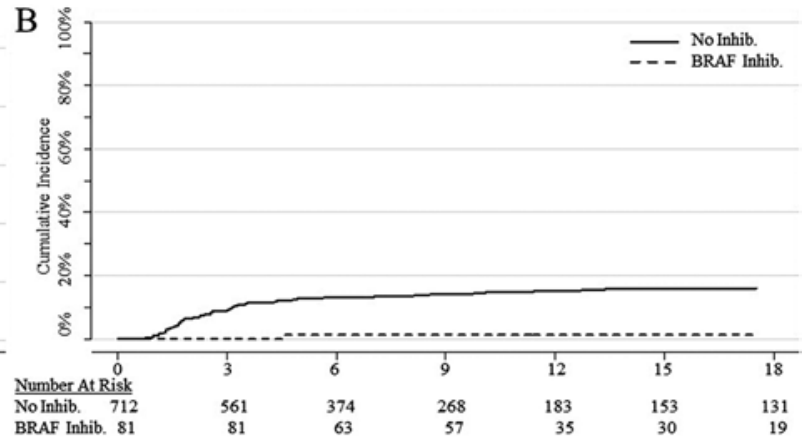

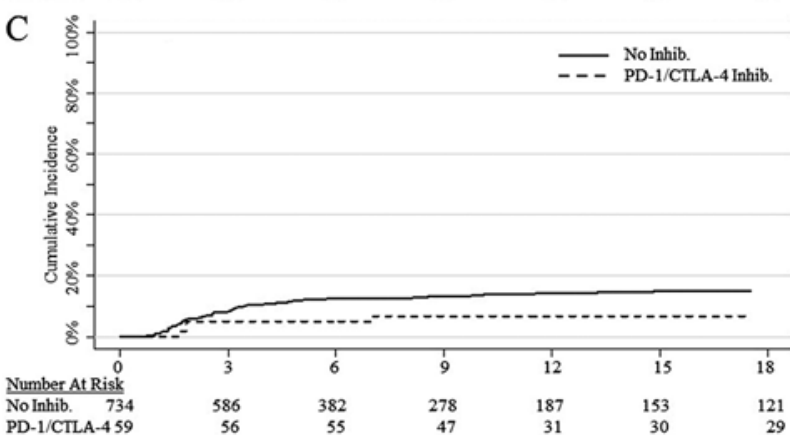

FIG. 2. Graphs showing cumulative incidences of LF for MBM undergoing SRS, stratified by (A) BRAF mutational status, (B) treatment with BRAFi, and (C) treatment with PD-1/CTLA-4 inhibitors.

605 (76\%) were $B R A F$-unknown. The median metastasis diameters were similar among patients with $B R A F^{m u t}$, $B R A F-W T$, and $B R A F$-unknown lesions $(0.9 \mathrm{~cm}$ vs 1.1 $\mathrm{cm}$ vs $1.1 \mathrm{~cm} ; \mathrm{p}=0.21$ ). Within 30 days of SRS, $73 \%$ of $B R A F^{m u t}$ metastases were exposed to BRAFi. In addition, $15 \%$ of BRAFmut $17 \%$ of BRAF-WT, and $5 \%$ of $B R A F-$ unknown metastases were exposed to immunotherapy (p $<0.01$ ), whereas $13 \%, 9 \%$, and $4 \%$, respectively, were exposed to cytokine therapy $(\mathrm{p}<0.01)$.

In this subset, $118(15 \%)$ LFs were observed among patients who received SRS. The 6- and 12-month cumulative incidences of LF were $12 \%(95 \%$ CI $10 \%-14 \%)$ and $14 \%$ (95\% CI 11\%-16\%), respectively. The 12-month cumulative incidence of LF was significantly lower among $B R A F^{m u t}$ lesions $(6 \%, 95 \%$ CI $2 \%-11 \%)$ compared with $B R A F-W T$ lesions $(22 \%, 95 \%$ CI $13 \%-32 \%$; $p<0.01$, Fig. $2 \mathrm{~A})$. For patients treated with upfront SRS alone, the 6- and 12-month cumulative incidences of DF were $48 \%(95 \%$ CI 39\%-58\%) and 69\% (95\% CI 59\%-78\%), respectively. The 12-month cumulative incidence of DF was $69 \%(95 \%$ CI 49\%-84\%) among BRAFmut lesions compared with $72 \%(95 \%$ CI 50\%-87\%) among BRAF-WT lesions ( $\mathrm{p}=$ 0.55 ). There was a significant reduction in the 12-month cumulative incidence of DF among patients with BRAFmut tumors who were treated with BRAFi after upfront SRS alone $(68 \%$ vs $95 \%, \mathrm{p}=0.03)$. Similarly, cytokines reduced the 12-month cumulative incidence from $73 \%$ to $47 \%$ (p $=0.03$ ). In contrast, immunotherapies did not appear to decrease the 12-month cumulative incidence of DF after upfront SRS alone ( $46 \%$ vs $44 \%, \mathrm{p}=0.40$ ).

Univariate analysis identified several variables associated ( $\mathrm{p} \leq 0.10)$ with LF, including age, number of brain metastases at presentation, $B R A F$ status, systemic therapies, prior surgery, prior WBRT, lesion size, and dosimetric pa- rameters (Table 3). On multivariate analysis, BRAF inhibition within 30 days of SRS was independently protective against LF (HR 0.08, 95\% CI 0.01-0.55; $p=0.01$ ). The 6and 12-month cumulative incidences of LF among lesions treated with BRAFi were both $1 \%(95 \%$ CI $1 \%-4 \%$ ) (Fig. 2B). Patients treated with immunotherapies also had a low risk of LF: the 6- and 12-month cumulative incidences were $5 \%$ (95\% CI 1\%-11\%) and 7\% (95\% CI 1\%-13\%) (Fig. 2C). Notably, immunotherapies were not protective against LF among patients with BRAF-WT lesions (HR $0.89,95 \%$ CI 0.13-3.99; $\mathrm{p}=0.40$ ).

Twenty-six patients developed RN in 45 lesions at a median of 3 months (range 2-53 months) after SRS. The 12-month cumulative incidences of RN were not significantly different among patients with $B R A F^{m u t}(3 \%, 95 \%$ CI $1 \%-6 \%), B R A F-W T(8 \%, 95 \%$ CI 2\%-14\%), and BRAFunknown tumors $(5 \%, 95 \%$ CI $3 \%-7 \%)(\mathrm{p}=0.18)$ (Fig. $3 \mathrm{~A})$. The 12-month cumulative incidences of $\mathrm{RN}$ were also low among lesions treated with BRAFi $(0 \%, 95 \%$ CI $0 \%-$ $0 \%$, immunotherapies $(2 \%, 95 \%$ CI $1 \%-5 \%)$, or cytokine therapies $(6 \%, 95 \%$ CI $1 \%-13 \%)$, and among lesions not exposed to targeted therapies (BRAF inhibitor-naive lesions) $(6 \%, 95 \%$ CI $4 \%-7 \%)(p=0.04$, Fig. 3B). Univariate analysis found that age, presence of extracranial metastases, $B R A F$ status, immunotherapy use, prior surgery, and dosimetric parameters were associated with development of RN (Table 3). Following multivariate analysis, $B R A F^{\text {mut }}$ status was protective (HR 0.27, 95\% CI 0.07-0.84; p = 0.02 ) against this complication.

\section{Discussion}

In the present study, we describe the clinical outcomes of more than 300 patients with MBM who were treated 

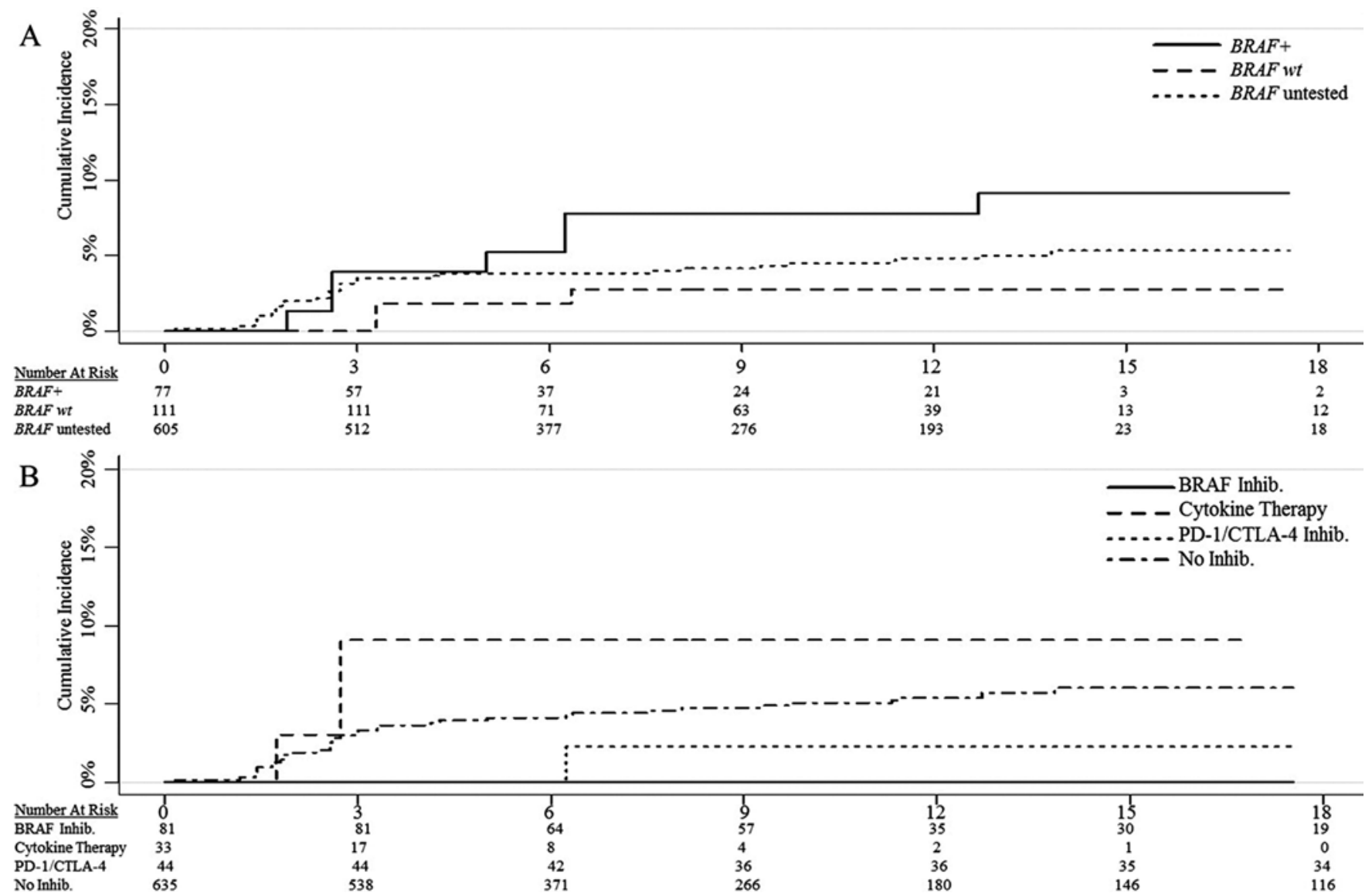

FIG. 3. Graphs showing cumulative incidences of RN for MBM treated with SRS, stratified by (A) BRAF status and (B) treatment with BRAFi and PD-1/CTLA-4 inhibitors.

for more than 1000 lesions, and we provide a lesion-based analysis of almost 800 MBMs treated with SRS. Our study resulted in several key findings that are important for clinical practice and trial design involving MBM. First, the clinical features at the time of presentation with brain metastasis differ in patients based on BRAF status. Second, there are several important patient and treatment factors to consider when estimating the survival of patients with MBM. Third, the use of BRAFi, immunotherapy, or cytokine therapy as an adjunct to radiation significantly improves survival for patients with MBM. Fourth, local control rates are high in patients with melanoma who were treated with SRS. Fifth, there appears to be an additive effect of SRS and BRAF directed therapies, with 1-year local control rates of $99 \%$. Sixth, patients with $B R A F^{m u t}$ lesions remain at low risk for $\mathrm{RN}$ following SRS, and treatment with targeted therapies does not significantly increase this complication.

The diagnosis-specific GPA for MBM identified only 2 prognostic variables for patient outcome: KPS score and number of brain metastases, with median survival times ranging from 3.4 to 13.2 months. ${ }^{29}$ In addition to these prognosticators, we investigated mutational status and the impact of targeted therapy or immunotherapy. The 6-month survival estimate for patients receiving targeted therapy or immunotherapies was approximately $75 \%$, compared with $50 \%$ for those who did not receive such treatments. Given these findings, it is important to consider inclusion of both $B R A F$ mutational status and the use of targeted therapy or immunotherapies into prognostic schema, because these patients may have extended survivals. In our cohort, 6 patients with $B R A F^{m u t}$ lesions (17\%) remained alive 3 years after diagnosis of MBM. Awareness of the contributors to extended survival may help us better estimate treatment outcome in the current era. Similar prognostic variables for survival were observed by Wolf et al., including KPS score, systemic disease status, number of brain metastases, and BRAF inhibitor status. ${ }^{31}$ However, other series have not corroborated these findings. ${ }^{16}$ It is important to note that patients whose disease has progressed while they receive BRAFi tend to have poor prognoses, similar to patients who have never received targeted treatment or immunotherapies. This finding is consistent with reports of acquired resistance to BRAFi with prolonged therapy, and inclusion of patients with $B R A F^{m u t}$ tumors who were treated with BRAFi at different times relative to radiotherapy may explain some of the heterogeneity in patient outcomes across studies.$^{30}$ Interestingly, in our series $B R A F$ mutational status alone was not prognostic of patient outcome, and prior studies evaluating the impact of mutational status have also demonstrated conflicting results. ${ }^{8,31} \mathrm{~A}$ multiinstitutional update of the diagnosis-specific GPA with consideration of molecular profiling data is warranted.

Given the adverse neurocognitive effects of WBRT 
and the lack of survival benefit with combined-modality therapy, patients are increasingly being treated with primary SRS. ${ }^{3,23}$ Earlier series of MBMs treated with SRS demonstrated local control rates $>75 \%$; however, these studies did not include stratification by $B R A F$ status and did not investigate the impact of treatment with BRAFi or immunotherapies. Preclinical models have demonstrated that subsets of melanoma cell lines are inherently radiosensitive and that there is a radiosensitization effect when BRAFi are used with combined-modality therapy in vitro. ${ }^{24}$ Interestingly, clinical experience has been mixed. Ly et al. reported improved local control for $B R A F^{m u t}$ lesions treated with BRAFi ( $85 \%$ vs 52\%, $\mathrm{p}=0.008)$, whereas Wolf et al. observed no significant difference in local control between patients with $B R A F^{\text {mut }}$ lesions being treated with BRAFi and those with BRAF-WT tumors (95\% vs $91 \%$, p $=0.51) \cdot{ }^{16,31}$ Overall, our series demonstrated favorable local control rates among all treated lesions (12-month rate: $86 \%)$. Overall, LF was significantly lower among $B R A F^{\text {mut }}$ lesions (12-month rate: 6\%), and the lowest LF was observed in patients treated with BRAFi within 4 weeks of SRS (12-month rate: 1\%). Furthermore, we observed no local recurrences when a BRAF inhibitor was given within 2 weeks of SRS.

The differences in the results across studies may be related to the timing of targeted therapy, specifically the interval between radiation and introduction of BRAFi. In the study by Ly et al., 54\% of lesions were not treated with a BRAF inhibitor within 4 weeks of SRS (i.e., before or after), and patients who had recently started or were about to start a BRAF inhibitor had a median washout period of 7 days (range 1-20 days). ${ }^{16}$ The possibility of a timing effect is also supported by a recent analysis of 75 patients with MBM treated with SRS, in which concurrent use (within 4 weeks of SRS) of immune checkpoint therapy was associated with a greater reduction in lesion volume up to 6 months posttreatment. ${ }^{21}$ The optimal timing of SRS in relation to the use of targeted and immune-based therapies requires additional investigation, but our results suggest a possible additive role with systemic therapy, related to the time of treatment.

Although BRAFi have demonstrated intracranial response rates of $39 \%-53 \%$ in untreated brain metastasis, it is important to note that patients treated with SRS remain at risk for DF.,14 This is especially important given that previous studies have determined that patients with melanoma (in addition to those with multiple lesions or uncontrolled extracranial disease) remain at the highest risk for DF after SRS. ${ }^{25}$ In our series, despite the impressively few LFs in patients with $B R A F^{\text {mut }}$ lesions, we continued to observe high rates of DF, which were partially reduced with the use of BRAFi and cytokines but not with immunotherapies. Intracranial tumor control remains the most important factor for survival with MBM. ${ }^{28}$ Therefore, studies should focus on combining SRS with medical therapies that have better CNS penetration or with combination therapies (e.g., a BRAF inhibitor concurrently with an MEK inhibitor) to help further improve patient outcomes. ${ }^{13}$

As the selection, sequencing, and combination of targeted therapies continue to evolve, we recommend frequent surveillance for patients treated with local modali- ties alone in the upfront or recurrent setting to monitor for intracranial progression. Although from our study we are not able to make specific treatment recommendations for patients at the time of distant intracranial failure based on mutational profile, important considerations include the patient's KPS score, number of brain metastases, size of the lesions, location of the lesions, neurological status of the patient, primary tumor extent and overall disease status, and the patient's input. We have previously shown, in a study of 59 patients who underwent a minimum of 3 courses of SRS to a total of 765 brain metastases, that this aggressive approach is associated with favorable intracranial control rates, modest treatment toxicity, does not negatively affect a patient's KPS score, and helps preserve a patient's quality of life. ${ }^{11}$ Therefore, consideration of repeated courses of SRS can be given to patients with MBM who have favorable risk factors, especially when additional systemic therapy options exist.

Safety reports on patients receiving BRAFi while undergoing SRS have primarily focused on cutaneous toxicities, given the early case reports of severe radiation dermatitis and cutis verticis gyrata in patients receiving concurrent WBRT and vemurafenib. ${ }^{10,26}$ Relatively few early complications have been reported in patients treated with targeted therapies and SRS. In a study of 24 patients undergoing SRS with concurrent vemurafenib, no significant adverse skin complications were observed. ${ }^{1}$ Gaudy-Marqueste et al. reported a $>20 \%$ increase in volume in $13.3 \%$ of brain lesions observed in patients who had received BRAFi after SRS; however, this did not correlate with neurological symptoms and was in fact higher in patients who stopped BRAFi compared with those who continued on the medication. ${ }^{7}$ There is limited information on late toxicity in patients treated with BRAFi and SRS. Ly et al. reported an increased hemorrhagic risk in patients treated with BRAFi compared with those treated with SRS alone (60\% vs $23 \%$ at 12 months); however, a study by Wolf and colleagues did not demonstrate a similar effect. ${ }^{16,31}$ In our series, RN rates were similar across $B R A F$ cohorts and low $(<5 \%)$ in patients who received either BRAFi or PD-1/CTLA-4 inhibitors. Therefore, given the impressive efficacy of combined-modality treatment, we consider the use of targeted agents within 4 weeks of SRS to be safe for clinical practice and optimal for tumor control. This is supported by recent consensus guidelines, which have recommended that BRAFi be withheld $\geq 1$ day before and after SRS. ${ }^{2}$ Given the low rates of $\mathrm{RN}$ in our series and lower local control rates when treating larger lesions, one could hypothesize that dose escalation or staged SRS sessions could provide a safe method of further improving local control rates.

There are several limitations to the present study, including its retrospective nature and the fact that patients tested for $B R A F$ represent a small number of our overall population. Moreover, the long period of the study (19872014) spans the development of many advances in diagnostic imaging and treatment options. Although this analysis does represent the largest lesion-based study of MBM, the small numbers of patients could have resulted in selection bias regarding those with $B R A F^{m u t}$ tumors, which may be reflected in the fact that this group had a longer time from primary diagnosis to brain metastasis and a higher inci- 
dence of SRS. These observations may be due to innate differences in the biology of the $B R A F$ mutation, or may simply include selection bias for treatments. Moreover, we classified all enlarging lesions as either an LF or RN by using our institutional algorithm. ${ }^{5}$ However, we did not report on the rates of pseudoprogression in this series, which we acknowledge could be useful among patients receiving BRAFi or checkpoint inhibitors, or other immunotherapies.

\section{Conclusions}

Historically, outcomes were poor in patients with MBMs. The use of SRS and the development of BRAFi and novel immunotherapies have changed the landscape of treatment options available to patients. Understanding the roles of each of these therapies, any possible synergy when used in combination, and their side effects is critical to improving outcomes and to providing evidence-based, personalized care for patients with MBM. The results of our study demonstrate that SRS along with BRAFi, immunotherapy, or cytokine therapy provide optimal local control in patients with MBM. Newer targeted and immune-based therapies, combination therapies to prevent acquired resistance, and optimized SRS techniques are key to further optimizing intracranial tumor control and patient outcomes.

\section{References}

1. Ahmed KA, Freilich JM, Sloot S, Figura N, Gibney GT, Weber JS, et al: LINAC-based stereotactic radiosurgery to the brain with concurrent vemurafenib for melanoma metastases. J Neurooncol 122:121-126, 2015

2. Anker CJ, Grossmann KF, Atkins MB, Suneja G, Tarhini AA, Kirkwood JM: Avoiding severe toxicity from combined BRAF inhibitor and radiation treatment: consensus guidelines from the Eastern Cooperative Oncology Group (ECOG). Int J Radiat Oncol Biol Phys 95:632-646, 2016

3. Brown PD, Asher AL, Ballman KV, Farace E, Cerhan JH, Anderson SK, et al: NCCTG N0574 (Alliance): A phase III randomized trial of whole brain radiation therapy (WBRT) in addition to radiosurgery (SRS) in patients with 1 to 3 brain metastases. J Clin Oncol 33 Suppl:LBA4, 2015 (Abstract)

4. Chang EL, Selek U, Hassenbusch SJ III, Maor MH, Allen PK, Mahajan A, et al: Outcome variation among "radioresistant" brain metastases treated with stereotactic radiosurgery. Neurosurgery 56:936-945, 2005

5. Chao ST, Ahluwalia MS, Barnett GH, Stevens GH, Murphy ES, Stockham AL, et al: Challenges with the diagnosis and treatment of cerebral radiation necrosis. Int J Radiat Oncol Biol Phys 87:449-457, 2013

6. Dummer R, Goldinger SM, Turtschi CP, Eggmann NB, Michielin O, Mitchell L, et al: Vemurafenib in patients with BRAF $^{\mathrm{V} 600}$ mutation-positive melanoma with symptomatic brain metastases: final results of an open-label pilot study. Eur J Cancer 50:611-621, 2014

7. Gaudy-Marqueste C, Carron R, Delsanti C, Loundou A, Monestier S, Archier E, et al: On demand Gamma-Knife strategy can be safely combined with BRAF inhibitors for the treatment of melanoma brain metastases. Ann Oncol 25:2086-2091, 2014

8. Gummadi T, Zhang BY, Valpione S, Kim C, Kottschade LA, Mittapalli RK, et al: Impact of BRAF mutation and BRAF inhibition on melanoma brain metastases. Melanoma Res 25:75-79, 2015
9. Hara W, Tran P, Li G, Su Z, Puataweepong P, Adler JR Jr, et al: Cyberknife for brain metastases of malignant melanoma and renal cell carcinoma. Neurosurgery 64 (2 Suppl):A26A32, 2009

10. Harding JJ, Barker CA, Carvajal RD, Wolchok JD, Chapman $\mathrm{PB}$, Lacouture ME: Cutis verticis gyrata in association with vemurafenib and whole-brain radiotherapy. J Clin Oncol 32:e54-e56, 2014

11. Kotecha R, Damico N, Miller JA, Suh JH, Murphy ES, Reddy CA, et al: Three or more courses of stereotactic radiosurgery for patients with multiply recurrent brain metastases. Neurosurgery [epub ahead of print], 2016

12. Liew DN, Kano H, Kondziolka D, Mathieu D, Niranjan A, Flickinger JC, et al: Outcome predictors of Gamma Knife surgery for melanoma brain metastases. Clinical article. J Neurosurg 114:769-779, 2011

13. Long GV, Stroyakovskiy D, Gogas H, Levchenko E, de Braud F, Larkin J, et al: Combined BRAF and MEK inhibition versus BRAF inhibition alone in melanoma. N Engl J Med 371:1877-1888, 2014

14. Long GV, Trefzer U, Davies MA, Kefford RF, Ascierto PA, Chapman PB, et al: Dabrafenib in patients with Val600Glu or Val600Lys BRAF-mutant melanoma metastatic to the brain (BREAK-MB): a multicentre, open-label, phase 2 trial. Lancet Oncol 13:1087-1095, 2012

15. Lwu S, Goetz P, Monsalves E, Aryaee M, Ebinu J, Laperriere $\mathrm{N}$, et al: Stereotactic radiosurgery for the treatment of melanoma and renal cell carcinoma brain metastases. Oncol Rep 29:407-412, 2013

16. Ly D, Bagshaw HP, Anker CJ, Tward JD, Grossmann KF, Jensen RL, et al: Local control after stereotactic radiosurgery for brain metastases in patients with melanoma with and without BRAF mutation and treatment. J Neurosurg 123:395-401, 2015

17. Margolin K, Ernstoff MS, Hamid O, Lawrence D, McDermott D, Puzanov I, et al: Ipilimumab in patients with melanoma and brain metastases: an open-label, phase 2 trial. Lancet Oncol 13:459-465, 2012

18. Miller JA, Bennett EE, Xiao R, Kotecha R, Chao ST, Vogelbaum MA, et al: Association between radiation necrosis and tumor biology after stereotactic radiosurgery for brain metastasis. Int J Radiat Oncol Biol Phys 96:1060-1069, 2016

19. Mingione V, Oliveira M, Prasad D, Steiner M, Steiner L: Gamma surgery for melanoma metastases in the brain. $\mathbf{J}$ Neurosurg 96:544-551, 2002

20. Mohammadi AM, Schroeder JL, Angelov L, Chao ST, Murphy ES, Yu JS, et al: Impact of the radiosurgery prescription dose on the local control of small $(2 \mathrm{~cm}$ or smaller) brain metastases. J Neurosurg 126:735-743, 2017

21. Qian JM, Yu JB, Kluger HM, Chiang VL: Timing and type of immune checkpoint therapy affect the early radiographic response of melanoma brain metastases to stereotactic radiosurgery. Cancer 122:3051-3058, 2016

22. Ramanujam S, Schadendorf D, Long GV: Systemic therapies for melanoma brain metastases: which drug for whom and when? Chin Clin Oncol 4:25, 2015

23. Sahgal A, Aoyama H, Kocher M, Neupane B, Collette S, Tago M, et al: Phase 3 trials of stereotactic radiosurgery with or without whole-brain radiation therapy for 1 to 4 brain metastases: individual patient data meta-analysis. Int J Radiat Oncol Biol Phys 91:710-717, 2015

24. Sambade MJ, Peters EC, Thomas NE, Kaufmann WK, Kimple RJ, Shields JM: Melanoma cells show a heterogeneous range of sensitivity to ionizing radiation and are radiosensitized by inhibition of B-RAF with PLX-4032. Radiother Oncol 98:394-399, 2011

25. Sawrie SM, Guthrie BL, Spencer SA, Nordal RA, Meredith $\mathrm{RF}$, Markert JM, et al: Predictors of distant brain recurrence for patients with newly diagnosed brain metastases treated 
with stereotactic radiosurgery alone. Int J Radiat Oncol Biol Phys 70:181-186, 2008

26. Schulze B, Meissner M, Wolter M, Rodel C, Weiss C: Unusual acute and delayed skin reactions during and after whole-brain radiotherapy in combination with the BRAF inhibitor vemurafenib. Two case reports. Strahlenther Onkol 190:229-232, 2014

27. Shaw E, Scott C, Souhami L, Dinapoli R, Kline R, Loeffler J, et al: Single dose radiosurgical treatment of recurrent previously irradiated primary brain tumors and brain metastases: final report of RTOG protocol 90-05. Int J Radiat Oncol Biol Phys 47:291-298, 2000

28. Sloan AE, Nock CJ, Einstein DB: Diagnosis and treatment of melanoma brain metastasis: a literature review. Cancer Contr 16:248-255, 2009

29. Sperduto PW, Kased N, Roberge D, Xu Z, Shanley R, Luo X, et al: Summary report on the graded prognostic assessment: an accurate and facile diagnosis-specific tool to estimate survival for patients with brain metastases. J Clin Oncol 30:419-425, 2012

30. Villanueva J, Vultur A, Lee JT, Somasundaram R, FukunagaKalabis M, Cipolla AK, et al: Acquired resistance to BRAF inhibitors mediated by a RAF kinase switch in melanoma can be overcome by cotargeting MEK and IGF-1R/PI3K. Cancer Cell 18:683-695, 2010

31. Wolf A, Zia S, Verma R, Pavlick A, Wilson M, Golfinos JG, et al: Impact on overall survival of the combination of BRAF inhibitors and stereotactic radiosurgery in patients with melanoma brain metastases. J Neurooncol 127:607-615, 2016

\section{Disclosures}

Dr. Suh received consulting payment from Varian Medical Systems; had travel and lodging paid for by Elekta; and received speaking fees from Philips. Dr. Ahluwalia received a consulting payment and grant from Elekta; grant support from Boehringer Ingelheim, Bristol-Myers Squibb, Novartis, Spectrum Pharmaceuticals, Tracon Pharmaceuticals, and Novocure; and is a consultant for Merck, Genentech/Roche, Incyte, Caris Lifesciences, Monteris Medical, MRI Interventions Inc., Bristol-Myers Squibb, Astrazeneca, and Abbvie. He received support of non-study-related clinical or research efforts that he oversaw from Novartis, Novocure, and Bristol-Myers Squibb. Dr. Kotecha is on the medical advisory board of Varian Medical Systems.

\section{Author Contributions}

Conception and design: Ahluwalia, Kotecha. Acquisition of data: Kotecha, Miller, Venur, Mohammadi. Analysis and interpretation of data: Ahluwalia, Kotecha, Miller, Chao, Funchain. Drafting the article: Ahluwalia, Kotecha, Miller, Funchain. Critically revising the article: Ahluwalia, Kotecha, Miller, Mohammadi, Chao, Suh, Barnett, Murphy, Funchain, Yu, Vogelbaum, Angelov. Reviewed submitted version of manuscript: Ahluwalia, Kotecha, Miller, Mohammadi, Chao, Suh, Barnett, Murphy, Yu, Vogelbaum, Angelov. Approved the final version of the manuscript on behalf of all authors: Ahluwalia. Statistical analysis: Kotecha, Miller.

\section{Supplemental Information}

\section{Previous Presentations}

Presented in part at the American Society of Clinical Oncology Annual Meeting in Chicago, Illinois, June 3-7, 2015. Presented in part at the American Society for Radiation Oncology 57th Annual Meeting in San Antonio, Texas, October 18-21, 2015.

\section{Correspondence}

Manmeet S. Ahluwalia, Department of Hematology/Oncology, Taussig Cancer Institute, Rose Ella Burkhardt Brain Tumor and Neuro-Oncology Center, Cleveland Clinic Main Campus, Mail Code S73, 9500 Euclid Ave., Cleveland, OH 44195. email: ahluwam@ccf.org. 